ORIGINAL

\title{
Diámetro del cuerpo lúteo y niveles de progesterona sérica, durante el ciclo estral en yeguas criollas colombianas
}

\section{Corpus luteum diameter and serum progesterone level, during the estrous cycle in colombian criollo mares}

\author{
Pilar Paredes $\mathrm{H},{ }^{1 *} \mathrm{MV}$, Claudia Jiménez $\mathrm{E}^{1}{ }^{1} \mathrm{Ph} . \mathrm{D}$, Aureliano Hernández $\mathrm{V}^{1} \mathrm{Ph} . \mathrm{D}$. \\ ${ }^{1}$ Universidad Nacional de Colombia, Facultad de Medicina Veterinaria y de Zootecnia, Centro de \\ Estudios de Postgrados. Bogotá D.C. Colombia. *Correspondencia: mdparedesh@unal.edu.co
}

Recibido: Mayo de 2012; Aceptado: Febrero de 2013.

\section{RESUMEN}

Objetivo. Determinar el diámetro del cuerpo lúteo (CL) y los niveles séricos de progesterona (P4) durante el ciclo estral (CE) en yeguas Criollas Colombianas (CC). Materiales y métodos. Se utilizaron 30 yeguas entre 4 a 14 años de edad, ubicadas en Pereira (Colombia). Se realizó ultrasonografía transrectal diariamente, desde la ovulación (día cero), durante dos CE para evaluar el diámetro del CL. Se tomaron muestras de sangre cada 48 horas entre una ovulación y la siguiente para cuantificar niveles séricos de P4 por la técnica de radioinmunoanálisis (RIA). Los datos fueron analizados con estadística descriptiva, desviación estándar, $t$ de Student para determinar diferencias entre los CE y una prueba de correlación entre el diámetro del CL y P4. Resultados. El tamaño del CL durante el CE fue $18.3 \pm 5.9 \mathrm{~mm}$ (promedio \pm desviación estándar). El diámetro el día cero fue $25.1 \pm 4.5$ $\mathrm{mm}$ y el tamaño final del CL $8.5 \pm 0.9 \mathrm{~mm}$ el día 20 del CE. El mayor diámetro se encontró el día 2 postovulación $(26.4 \pm 5.0 \mathrm{~mm})$. Los niveles máximos de $\mathrm{P} 4$ se encontraron al día 6 postovulación $(10.7 \pm 4.3$ rango 2.51 a $18.8 \mathrm{ng} / \mathrm{ml})$. La concentración de P4 durante el diestro fue $6.6 \pm 3.6(1.15 \mathrm{a}$ $10.7 \mathrm{ng} / \mathrm{ml}$ ) y durante el estro $0.25 \pm 0.3(0.01 \mathrm{~m}$ a $0.86 \mathrm{ng} / \mathrm{ml})$. Conclusiones. La yegua CC presenta una dinámica del CL similar a la reportada en la literatura. Los valores aquí reportados pueden ser el punto de partida para establecer valores de referencia de utilidad clínica.

Palabras clave: Muestreo sanguíneo, ovulación, ultrasonografía, yeguas (Fuente: $C A B$ ). 


\section{ABSTRACT}

Objective. To determine the corpus luteum (CL) diameter and serum progesterone levels (P4) during the estrous cycle (EC) in Colombian Criollo mares (CC). Materials and methods. Thirty 4 to 14-years old mares were employed. The animals were located in Pereira (Colombia). Transrectal ultrasonography was performed daily through two EC, from ovulation (day zero) to the next ovulation. Blood samples were taken every 48 hours during one EC, to measure serum levels of P4 by radioimmunoassay (RIA). Data analysis was performed using descriptive statistics, with standard deviation, and student's $\mathrm{t}$-distribution to determine differences between the EC and a test of correlation between CL diameter and P4 levels. Results. The size of the CL during the EC was $18.3 \pm 5.9 \mathrm{~mm}$ (mean \pm standard deviation). The average $C L$ diameter found on day zero was $25.1 \pm 4.5 \mathrm{~mm}$ and the final size, was $8.5 \pm 0.9 \mathrm{~mm}$ on day 20 of the EC. The larger CL diameter was found on day 2 post-ovulation $(26.4 \pm 5.0 \mathrm{~mm})$. The highest serum P4 levels were found on day 6 post-ovulation ( $10.7 \pm 4.3$ range from 2.51 to $18.8 \mathrm{ng} / \mathrm{ml})$; during diestrus, they were $6.6 \pm 3.6$ (range 1.15 to $10.7 \mathrm{ng} / \mathrm{ml}$ ) and during estrus, $0.25 \pm 0.3$ (range 0.01 to $0.86 \mathrm{ng} / \mathrm{ml}$ ). Conclusions. The CC mare $\mathrm{CL}$ presents a dynamic similar to that reported in the literature. Present findings could be used as a starting point for the establishment of reference values of clinical importance.

Key words: Blood sampling, mares, ovulation, ultrasonography (Source: $C A B$ ).

\section{INTRODUCCIÓN}

La ovulación, sucede en gran parte, como respuesta del folículo dominante al aumento en los niveles circulantes de la hormona luteinizante (LH) e involucra eventos encadenados que terminan en la ruptura del mismo; ocurre en promedio 4.2 días después que el folículo dominante alcanza $35 \mathrm{~mm}$ de diámetro (1). Cuando el folículo se rompe durante la ovulación también hay ruptura de vasos sanguíneos de la pared folicular, esto lleva a la formación de una cavidad que contiene fluido folicular y sangre proveniente de la ovulación, la cual es conocida como cuerpo hemorrágico $(\mathrm{CH})$. En condiciones normales, el $\mathrm{CH}$ puede ser detectado desde el momento de la ovulación hasta 1 a 3 días postovulación, posteriormente comienza a aumentar su tamaño y a perder su apariencia hemorrágica transformándose en cuerpo lúteo (CL) $(1,2)$. Después de la ovulación, ocurre la luteinización en las células de la teca interna y las células de la granulosa del folículo; en este proceso las células del folículo ovulatorio se convierten en tejido luteal (1).

La maduración funcional del CL está caracterizada por un aumento progresivo de las concentraciones circulantes de progesterona (P4) $(>1 \mathrm{ng} / \mathrm{ml})$, lo que se relaciona estructuralmente con un aumento en el diámetro del $\mathrm{CL}$, incremento de la irrigación sanguínea y disminución de la ecogenicidad $(1,2)$. Las concentraciones séricas máximas de $\mathrm{P} 4$ durante la mitad del diestro (días 5 a 7) pueden estar entre 4 y $10 \mathrm{ng} / \mathrm{ml}$ (3) y se correlacionan con un mayor diámetro luteal, con un aumento de la irrigación sanguínea y con un nivel de baja ecogenicidad. Los niveles séricos de P4 permanecen elevados por un período de
6 a 10 días y declinan rápidamente hasta llegar a niveles basales $(1,3)$. Posterior al día 13-16 del diestro (diestro tardío), la prostaglandina F2alfa (PGF2a) se sintetiza en el endometrio, alcanza el ovario por vía sistémica y genera la regresión funcional del $\mathrm{CL}$, caracterizada por una disminución progresiva de los niveles de P4, del diámetro luteal y de la vascularidad, y por un aumento en la ecogenicidad (1). Durante el estro, las concentraciones de $\mathrm{P} 4$ son menores de $1 \mathrm{ng} / \mathrm{ml}(3,4)$.

En un estudio en yeguas criollas colombianas (CC) ubicadas en Bogotá, se evaluaron los niveles de P4 cada tercer día, encontrando valores de producción de P4 más altos que los reportados para las demás razas. La producción máxima de P4 se situó entre los 9.35 y $28.57 \mathrm{ng} / \mathrm{ml}$; a partir del día 9 comenzó la caída de la curva de P4 y en el día 15 sus niveles se acercaron a $1 \mathrm{ng} /$ ml (5). En España en un estudio con dos razas de yeguas, se concluyó que las concentraciones de P4 plasmática no manifiestan diferencias significativas entre los dos genotipos durante el celo, pero sí en la fase luteal; los valores en la raza árabe oscilaron entre 5.98 a 12.8 mientras que para la raza andaluza variaron entre 4.58 a $8.3 \mathrm{ng} / \mathrm{ml}(6)$.

Dada la amplia variación de las condiciones climáticas en el trópico colombiano y su conocida injerencia en la función ovárica de la yegua, se hace necesario establecer los valores de referencia de la fisiología reproductiva de importancia clínica en yeguas CC. Lo anterior permitiría mejorar y aumentar la eficiencia reproductiva de cada animal y tomar las medidas 
preventivas y curativas correspondientes según el caso y para futuros estudios que contribuyan a la preservación de la raza CC.

El presente trabajo tuvo como objetivo establecer los cambios en los niveles séricos de P4 durante un CE; determinar la evolución del desarrollo y medir el CL por medio de ultrasonografía durante dos CE consecutivos en yeguas CC.

\section{MATERIALES Y MÉTODOS}

Animales y condiciones medioambientales. Se utilizaron 30 yeguas de la raza CC ubicadas en diferentes zonas rurales del municipio de Pereira (Risaralda), a una altura de $1.411 \mathrm{msnm}$, $4^{\circ} 49^{\prime}$ de latitud norte y $75^{\circ} 42^{\prime}$ de longitud oeste; temperatura promedio de $21.8^{\circ} \mathrm{C}$, humedad relativa de $75 \%$, precipitación media de 2.210 $\mathrm{mm} / \mathrm{año} \mathrm{y}$ un promedio de brillo solar diario de 13 horas (7). Lo anterior corresponde al llamado piso térmico templado colombiano (8).

La edad de los animales osciló así: entre los 4 a 7 años $(n=17)$, de 8 a 11 años $(n=10)$ y de 12 a 14 años $(n=3)$. El peso corporal, entre 320 y $400 \mathrm{Kg}$, con una buena condición corporal (en promedio 6/9). Todos los animales permanecieron estabulados, con una dieta de pasto de corte King grass (Pennisetum purpureum), el cual se suministró a voluntad, $3 \mathrm{~kg}$ de concentrado al día (12-15\% nivel de proteína) y sal a voluntad. Las yeguas se hallaban en buenas condiciones de salud y sin ninguna anormalidad reproductiva, lo cual fue determinado por medio de la inspección visual de los órganos reproductivos externos, palpación rectal y ultrasonografía del tracto reproductivo. Se utilizó un ecógrafo Honda electronics modelo HS $1500 \mathrm{~V}$ (Honda Electronics Co., Ltd, Japón), equipado con un transductor rectal lineal multifrecuencia de 10, 7.5 y $5 \mathrm{MHz}$ modelo HLV-375M y con capacidad de almacenamiento de 60 imágenes. Se empleó una sonda de 7.5 Mhz.

Seguimiento ultrasonográfico. El presente trabajo se inició al identificar por ultrasonografía el día de la ovulación, el cual se tomó como el día cero. A partir de este momento se comenzó un seguimiento ecográfico diario de cada yegua, durante dos CE consecutivos, para determinar el diámetro del $C L$, el cual se obtuvo del promedio al medir el CL transversal y longitudinalmente. En el $\mathrm{CH}$ se realizó la medición tomando desde el borde interno de la pared.

Cuantificación de los niveles plasmáticos de P4. Se tomaron muestras de sangre por medio de punción de la vena yugular con el sistema de vacutainer ${ }^{\circledR}$ en tubos sin anticoagulante, los días $0,2,4,6,8,10,12,14,16,18$ y 20 de uno de los CE hasta la siguiente ovulación. Este muestreo se realizó durante el seguimiento de uno de los dos CE en cada yegua seleccionada y la muestra obtenida se centrifugó a 3000 r.p.m. durante 15 minutos, para obtener el suero sanguíneo, que fue almacenado en viales plásticos a $-20^{\circ} \mathrm{C}$. La cuantificación de los niveles de P4 se realizó por la técnica de RIA de fase sólida con el kit comercial Coat-A-Count Progesterona ${ }^{\circledR}$ (Siemens, USA), con una especificidad de $0,02 \mathrm{ng} / \mathrm{ml}$. A partir de los niveles de P4 se caracterizaron las fases del CE de la yegua CC; donde los valores inferiores a $1 \mathrm{ng} / \mathrm{ml}$ indicaron la fase folicular, y los valores superiores a este valor determinaron la fase luteal.

Análisis estadístico. Se aplicó la estadística descriptiva con valores de promedio y desviación estándar (DE) para todos los parámetros a evaluar. Se realizó una prueba $t$ de Student entre los datos del primer y segundo CE para determinar si existían diferencias, y entre los diámetros del $\mathrm{CL}$ de los dos CE. También se realizó una prueba de correlación de Pearson entre el diámetro del CL y los niveles de P4 sérica.

\section{RESULTADOS}

De acuerdo con los niveles de P4 y los hallazgos ultrasonográficos, la duración promedio del primer CE fue de $21.7 \pm 2.3$ (rango 18 a 28 días), con una fase luteal de $14.2 \pm 1.4$ días (rango 11-17 días) y de 6.7 \pm 2.2 días (rango 3-12 días) para la fase folicular. En el segundo CE la duración promedio fue de $21.7 \pm 2.8$ (rango 16 a 33 días), con una fase luteal de $14.3 \pm 1.0$ días (rango 13-17 días) y de 7.6 5.0 días (rango 3-17 días) para la fase folicular.

Desarrollo del cuerpo lúteo durante el ciclo estral. Aunque no se encontraron diferencias entre los promedios del diámetro del CL de los dos CE analizados, los resultados obtenidos de cada uno de ellos, se presentan por separado en las tablas 1 y 2. En el día cero el $\mathrm{CH}$ hiperecogénico tenía una cavidad intraluteal llena de fluido, la cual alcanzó su mayor tamaño el día 2 postovulación (26.4 45.0 $\mathrm{mm}$ ) (promedio $\pm \mathrm{DE}$ ). El diámetro promedio encontrado el día cero fue de $25.1 \pm 4.5 \mathrm{~mm}$ y el tamaño final del CL fue de $8.5 \pm 0.9 \mathrm{~mm}$ el día 20 del CE. El diámetro promedio del CL durante el CE fue de $18.3 \pm 5.9$ con un rango de 7.3 a $26.4 \mathrm{~mm}$ (última y primera observación, respectivamente) (Figura 1). El CL disminuyó progresivamente de tamaño y se pudo visualizar, en promedio hasta el día $16.0 \pm 2.0$ (11 a 20 días postovulación). 


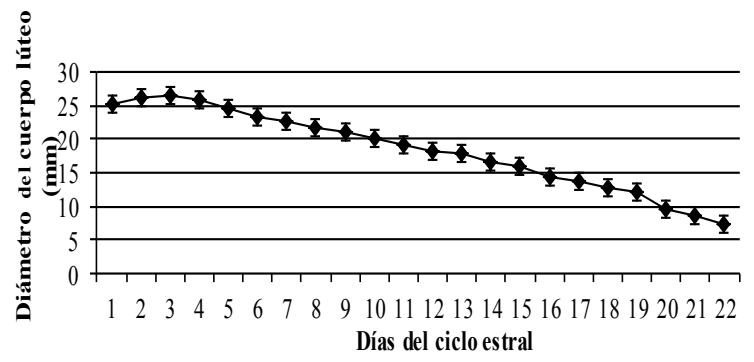

Figura 1. Valor promedio y desviaciones estándar del diámetro del cuerpo lúteo durante el ciclo estral de yeguas criollas colombianas $(n=30)$.

Entre los días 0 y 4 del primer CE observado, los valores promedio de los rangos de los $\mathrm{CL}$ analizados fueron $24.70-25.88 \mathrm{~mm}$ y para el segundo CE de $26.19-27.50 \mathrm{~mm}$. Entre los días 4 y 8 , los valores correspondientes fueron $21.57-$ $22.86 \mathrm{~mm}$ y $22.94-24.26$, respectivamente. Para los días 8 a 12 fueron de 18.59 - 19.69 mm y 19.1-21.06 mm, respectivamente. Para los días 12 a 16 , fueron $15.29-16.85$ y $15.41-17.23$ respectivamente. Después del día 16 del CE los valores estuvieron entre $10.5-12.68$, y $11.93-$ $12.50 \mathrm{~mm}$ (Figura 2, tablas 1 y 2). La imagen ultrasonográfica del CL en diferentes días del CE puede ser observada en la figura 3.

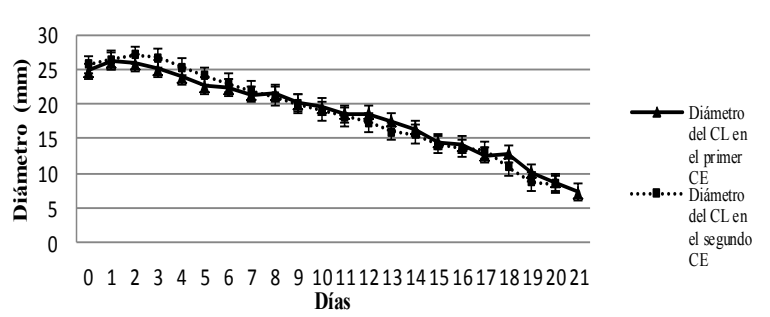

Figura 2. Valor promedio y desviaciones estándar del diámetro del cuerpo lúteo durante los dos ciclos estrales consecutivos de yeguas criollas colombianas $(n=30)$.

Tabla 1. Valores promedio del diámetro del cuerpo lúteo $(\mathrm{mm})$ en yeguas criollas colombianas $(n=29 *)$ durante el primer ciclo estral

\begin{tabular}{cccccc}
\hline $\begin{array}{c}\text { Porcentaje de } \\
\text { yeguas en cada } \\
\text { rango }\end{array}$ & día 0-4 & día 4-8 & día 8-12 día 12-16 día 16... \\
\hline $0-20 \%$ & 25.88 & 21.57 & 19.47 & 16.47 & $10.5 \pm$ \\
& \pm 5.36 & \pm 0.79 & \pm 1.87 & \pm 1.06 & 0.11 \\
$20-40 \%$ & 24.40 & 21.08 & 18.59 & $15.29 \pm$ & $9.65 \pm$ \\
& \pm 2.09 & \pm 0.69 & \pm 1.24 & 1.66 & 1.20 \\
$40-60 \%$ & 24.63 & 21.86 & 19.13 & $15.91 \pm$ & $10.59 \pm$ \\
& \pm 1.53 & \pm 1.42 & \pm 1.28 & 1.59 & 1.84 \\
$60-80 \%$ & 24.68 & 22.24 & 19.34 & $16.50 \pm$ & $13.1 \pm$ \\
& \pm 1.08 & \pm 1.40 & \pm 1.13 & 1.75 & 5.24 \\
$80-100 \%$ & 24.70 & 22.86 & 19.69 & $16.85 \pm$ & $12.68 \pm$ \\
& \pm 0.97 & \pm 1.83 & \pm 1.25 & 1.71 & 4.63 \\
\hline
\end{tabular}

*Se excluyeron los datos de una yegua que presentó ovulación doble.
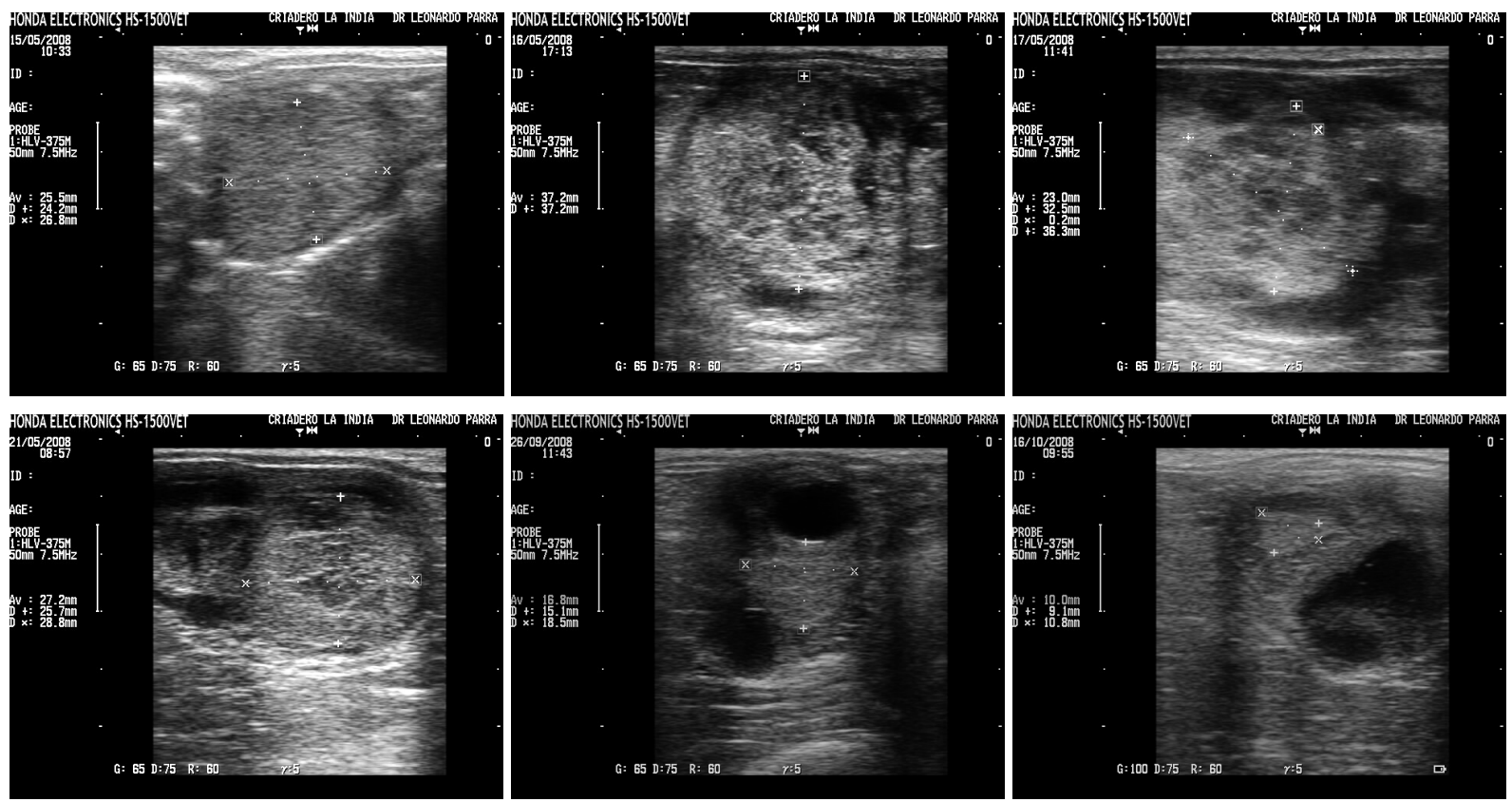

Figura 3. Imagen ultrasonográfica del cuerpo lúteo en diferentes días del ciclo estral en yeguas criollas colombianas. Cuerpo lúteo observado el día 0 (A), día 1 (B), Día 2(C), día 6 (D), día 14 (E) y día 19 (F) del ciclo estral (imágenes provenientes de diferentes animales). 
Tabla 2. Valores promedio del diámetro del cuerpo lúteo en yeguas criollas colombianas $(n=30)$ durante el segundo ciclo estral.

\begin{tabular}{cccccc}
\hline $\begin{array}{c}\text { Porcentaje de } \\
\text { yeguas en cada día 0-4 } \\
\text { rango }\end{array}$ & día 4-8 & día 8-12 día 12-16 día 16... \\
\hline $0-20 \%$ & 27.50 & 24.26 & 21.06 & 17.23 & 11.93 \\
& \pm 1.03 & \pm 0.38 & \pm 0.42 & \pm 0.55 & \pm 0.17 \\
$20-40 \%$ & 26.45 & 23.35 & 19.44 & 15.71 & 12.44 \\
& \pm 1.48 & \pm 1.27 & \pm 2.29 & \pm 2.15 & \pm 0.71 \\
$40-60 \%$ & 26.38 & 23.37 & 19.53 & 16.02 & 12.43 \\
& \pm 1.05 & \pm 0.90 & \pm 1.63 & \pm 1.61 & \pm 0.50 \\
$60-80 \%$ & 26.07 & 22.94 & 19.1 & 15.63 & 12.50 \\
& \pm 1.06 & \pm 1.13 & \pm 1.58 & \pm 1.53 & \pm 0.44 \\
$80-100 \%$ & 26.19 & 23.06 & 19.11 & 15.41 & 12.02 \\
& \pm 0.96 & \pm 1.02 & \pm 1.37 & \pm 1.41 & \pm 1.15 \\
\hline
\end{tabular}

De las 30 yeguas analizadas solamente una presentó ovulación doble; los datos de esta yegua se excluyeron del análisis de diámetro de CL y de la P4 durante el CE. En la yegua con ovulación doble el diámetro promedio encontrado el día cero fue de $21.7 \pm 2.1 \mathrm{~mm}$ (20.2 mm y $23.3 \mathrm{~mm}$ el CL del ovario derecho e izquierdo, respectivamente) y el tamaño final del CL del ovario derecho fue $13.8 \mathrm{~mm}$ el día 17 del CE y el del ovario izquierdo $11.4 \mathrm{~mm}$ el día 18 del CE; el CL alcanzó su mayor tamaño el día 2 postovulación ( $27.7 \mathrm{~mm}$ en el ovario derecho y $25.5 \mathrm{~mm}$ en el izquierdo). El diámetro promedio del CL durante el CE en la yegua con ovulación doble fue de $18.8 \pm 4.1$ con un rango de 11.4 a $26.6 \mathrm{~mm}$ (última y primera observación, respectivamente).

Cuantificación de los niveles séricos de progesterona (P4) durante el ciclo estral. En promedio entre el día 0 y 16 del CE los valores de P4 fueron superiores a $1 \mathrm{ng} / \mathrm{ml}$. El día cero (día de la ovulación) fue de $1.8 \pm 2.9 \mathrm{ng} / \mathrm{ml}$ (rango 0.003 a $12.2 \mathrm{ng} / \mathrm{ml}$ ) y posteriormente incrementó hasta alcanzar el valor máximo hacia el día 6 postovulación, de $10.7 \pm 4.3$ con un rango de 2.51 a $18.8 \mathrm{ng} / \mathrm{ml}$. Los niveles permanecieron elevados por 6 a 12 días $(9.6 \pm 1.1 \mathrm{ng} / \mathrm{ml})$ y a partir de este momento comenzó la caída drástica de la curva de P4 hasta llegar a niveles inferiores a $1 \mathrm{ng} / \mathrm{ml}$. La concentración de P4 durante el diestro fue de $6.6 \pm 3.6$ con un rango de 1.15 a $10.7 \mathrm{ng} / \mathrm{ml}$ y

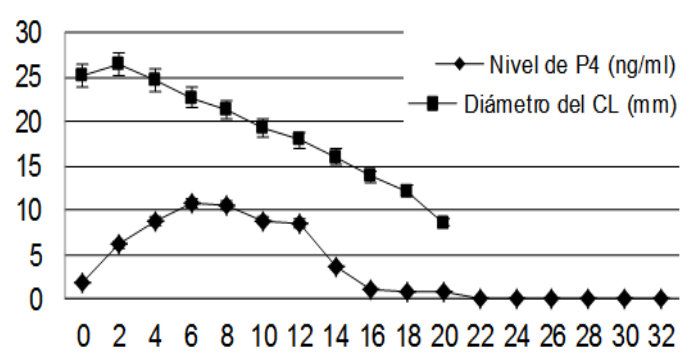

Figura 4. Valores promedio y desviación estándar de los niveles séricos de progesterona $(\mathrm{ng} / \mathrm{ml})$ y del diámetro del cuerpo lúteo durante el ciclo estral de yeguas criollas colombianas $(n=30)(r=0.58)$. durante el estro de $0.25 \pm 0.3$ con un rango de 0.01 a $0.86 \mathrm{ng} / \mathrm{ml}$. En la figura 4 se puede observar la variación de los valores obtenidos para los niveles séricos de $\mathrm{P} 4$ y el diámetro del CL durante el CE. No se encontró una correlación entre el diámetro del CL y los niveles séricos de P4 $(r=0.58)$. El coeficiente de variación intraensayo fue de $2.09 \%$. Los niveles séricos de $\mathrm{P} 4$ fueron más altos en la yegua con ovulación doble, en comparación con los de las otras yeguas (Figura 5 )

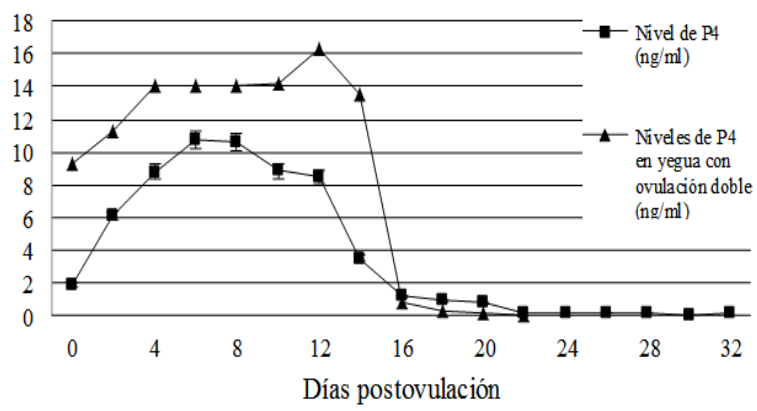

Figura 5. Valores promedio de los niveles séricos de progesterona $(\mathrm{ng} / \mathrm{ml})$ durante el ciclo estral de yeguas criollas colombianas $(n=30)$ comparados con una yegua que presentó ovulación doble $(p<0,05)$. Las barras de error representan la desviación estándar.

\section{DISCUSIÓN}

Dado que el CL en la yegua no es detectable por medio de palpación rectal, la ultrasonografía es una herramienta muy útil y precisa para determinar si ocurre la ovulación, evaluar la morfología del CL y su permanencia así como para establecer la relación entre estructura y función asociada con el desarrollo folicular y luteal $(3,9)$. Esta información es útil ya que se ha descrito que la actividad luteal disminuida o prolongada está asociada con patologías uterinas como endometritis o piómetra los cuales pueden llevar a la liberación prematura o disminución de PGF2 alfa (1).

En el presente estudio, no se encontraron diferencias entre los promedios del diámetro del CL durante los dos CE analizados. Esto implica que posiblemente hay una regularidad en el desarrollo del CL en la yegua durante CE sucesivos (aunque es deseable que haya más estudios al respecto). Por ende, los valores aquí reportados, pueden tener importancia como referencia en las evaluaciones clínicas para definir posibles anomalías como por ejemplo insuficiencias luteales, que pueden resultar en pérdidas embrionarias; además se ha descrito que los cambios en la morfología del $C L$, evaluados mediante ultrasonografía, reflejan modificaciones 
estructurales asociados con el desarrollo funcional del CL $(1,2)$. El diámetro promedio del CL en este estudio fue menor ( $18.3 \pm 5.9 \mathrm{~mm}$ ) comparado con el diámetro reportado en un estudio en yeguas Pura Sangre Inglés $(25.3 \pm 3.8 \mathrm{~mm})(10)$, lo cual puede indicar una diferencia del diámetro del $\mathrm{CL}$ según la raza y el tamaño del animal. Los datos aquí obtenidos acerca de los rangos para el diámetro del $\mathrm{CL}$, tienen pertinencia en los análisis clínicos si no hay la posibilidad de obtener información acerca de los valores séricos de P4. Es recomendable corroborar los datos obtenidos en este estudio, ya sea en el mismo genotipo equino o en otros.

En el presente estudio se encontraron concentraciones séricas de P4 durante el CE de la yegua, con niveles superiores durante la fase luteal $(6.6 \pm 3.6 \mathrm{ng} / \mathrm{ml})$ comparado con la fase folicular $(0.25 \pm 0.3 \mathrm{ng} / \mathrm{ml})$ como se describió en diferentes estudios $(3,4)$. Las concentraciones de $\mathrm{P} 4$ fueron más altas en la mitad de la fase luteal que al comienzo de ésta; el nivel máximo de $\mathrm{P} 4$ se encontró el día 6 postovulación, lo cual se debe a la maduración del CL que alcanza su máxima capacidad funcional en este momento (1). Se han reportado valores de $\mathrm{P} 4$ en yeguas durante el diestro entre 6 y $10 \mathrm{ng} / \mathrm{ml}$ (4); en un estudio en yeguas $\mathrm{CC}$ los valores de $\mathrm{P} 4$ encontrados en el punto máximo están entre 9.35 y $28.57 \mathrm{ng} / \mathrm{ml}$ (5). En el presente estudio, la concentración de P4 durante la fase luteal fue de $6.6 \pm 3.6$ con un rango de 1.15 a $10.7 \mathrm{ng} / \mathrm{ml}$ y el rango del nivel encontrado en el día de máxima concentración de P4 estuvo entre 2.51 a $18.8 \mathrm{ng} / \mathrm{ml}$.

En estudios morfológicos del $\mathrm{CL}$ se ha encontrado que éste presenta un área vascular de mayor tamaño durante el diestro temprano y en la mitad del diestro comparado con el diestro tardío; además que los niveles de P4 sérica han sido más altos cuando hay un $\mathrm{CL}$ maduro, el cual tiene un área vascular más grande y un mayor número de vasos sanguíneos (11). Este aumento en la irrigación sanguínea en el CL maduro y en la secreción de P4 también se reporta en otras especies $(12,13)$. Esto puede explicar por qué en el presente estudio se encontró un mayor diámetro al inicio de la fase luteal que coincidió con la maduración del $\mathrm{CL}$ y los niveles de $\mathrm{P} 4$ sérica más altos en la mitad de la fase luteal; y al final disminuyó el diámetro del CL y los niveles de P4 coincidiendo con la luteólisis.

En el presente estudio, entre el día 6 y 14 del CE no se encontró una correlación entre el diámetro del $C L$ y los niveles séricos de P4 $(r=0.58)$. En algunos estudios se ha encontrado una relación paralela entre la disminución del área del CL y las concentraciones de P4 durante la luteólisis (14); sin embargo no se han encontrado diferencias en la producción de $\mathrm{P} 4$ al compararla entre dos tipos diferentes de $\mathrm{CL}$, con y sin área central anecogénica y tampoco se encontró una relación entre el tamaño del CL y la producción de P4 en yeguas receptoras (15), lo cual puede sugerir que no hay una correlación entre el diámetro medido por ultrasonografía y los niveles séricos de P4 (16).

La duración del diestro en las yeguas puede ser de 14 a 15 días (4); en las razas Árabe y Andaluz se reportó una duración del diestro de $16.5 \pm 2.25$ días (6) y en yeguas criollas colombianas ubicadas en Bogotá $13.9 \pm 1.52$ días (5). En el presente estudio la duración de la fase luteal fue de $14.2 \pm 0.07$ días, lo cual coincide con lo descrito en la literatura. Es posible que las variaciones individuales influyan en la duración del diestro, pues la correspondiente al CE tiene fluctuaciones que son consideradas normales, lo cual es válido para las diversas fases del CE.

En el presente estudio se encontró solamente una yegua con ovulación doble, que tuvo los niveles séricos de P4 durante el CE más altos de todos los animales incluidos en el estudio. Las yeguas que presentan ovulación doble tienen aumentos significativos en los niveles de P4 sérica, 24 horas después de la segunda ovulación (4). La incidencia de ovulación múltiple aumenta de manera importante en yeguas de mayor edad, además de estar influenciada por la raza (Pura Sangre Inglés, Caballos de tiro y Trotones), el estado reproductivo (yeguas primerizas, yeguas en lactancia) y el estado nutricional (17). En el presente estudio, la yegua que presentó ovulación múltiple tenía 4 años de edad y no se encontraba bajo la influencia de ninguno de los factores mencionados anteriormente (datos que no se incluyen).

En conclusión los niveles séricos de P4 en la yegua CC son similares a los descritos en la literatura en otras razas y para países estacionales o en diferentes zonas del trópico colombiano. Los cambios en la estructura y forma del CL reflejan su desarrollo funcional, por lo cual se considera importante evaluarlo morfológicamente para diagnosticar posibles anormalidades, como complemento a la cuantificación de los niveles séricos de P4.

La variación de los resultados en cuanto a diámetro del CL y niveles séricos de P4 es muy amplia; por esto siempre se debe recordar la individualidad en el comportamiento reproductivo, porque existen factores genéticos, medioambientales y nutricionales que pueden influenciar y hacer variar la fisiología reproductiva. Por esto es importante realizar seguimientos más detallados y continuos, llevar registros individuales para conocer las características fisiológicas de cada animal.

Es necesario realizar más estudios en yeguas $\mathrm{CC}$ en diferentes condiciones medioambientales para establecer los factores que podrían afectar el 
funcionamiento reproductivo de cada una de las yeguas; además sería importante evaluar otras hormonas involucradas durante el CE y estimar el área de las células del $\mathrm{CL}$, los receptores para $\mathrm{LH}$ en las mismas y posibles correlaciones con el peso y diámetro del CL. Los valores aquí reportados pueden ser el punto de partida para el establecimiento de valores de referencia de utilidad clínica.

\section{Agradecimientos}

A la Universidad Nacional de Colombia por la financiación parcial de esta investigación. Al Laboratorio de hormonas de la Universidad Nacional de Colombia por permitir el procesamiento y lectura de las muestras. A Fedequinas por la ayuda en la financiación de este trabajo. A los diferentes criaderos equinos que prestaron sus animales para este estudio.

\section{REFERENCIAS}

1. Bergfelt DR and Adams GP. Current Therapy in Equine Reproduction, Missouri: Saunders Elsevier, 2007; 1-13.

2. Newcombe JR. Ultrasonography of ovulation and development of the corpus luteum in the mare. Equine Vet Ed 1996; 8(1):47-58.

3. Nett TM, Pickett BW, Seidel GE, Voss JL. Levels of luteinizing hormone and progesterone during the estrous cycle and early pregnancy in mares. Biol Reprod 1976; 14:412-415.

4. Daels PF, Hughes JP. McKinnon AO: Equine reproduction, Philadelphia: Lea \& Febiger; 1993.

5. López JJ, Rodríguez CA, Atuesta JE y Grajales HA. Nota sobre la dinámica ovárica y niveles de progesterona durante el ciclo estral de yeguas Criollas colombianas en la sabana de Bogotá-Colombia. Livest Res Rural Dev 2008; 20(10):167.

6. Vivo R, Santisteban R, Tovar P, Castejon F. Valores de progesterona en plasma de yeguas españolas y árabes durante el ciclo reproductor. Archivos de Zootecnia 1986; 35(131):59-67.

7. Ideam, Instituto de Hidrología, Meteorología y estudios ambientales de Colombia [Internet]. Colombia: Centro de documentación e Información científico técnica; 2005. Atlas Climatológico de Colombia. Parte III. [revisado 2007 jun 01]. URL Disponible en: https: //documentacion. ideam. gov.co/ openbiblio/ Bvirtual/019711.htm

8. Ideam, Instituto de Hidrología, Meteorología y estudios ambientales de Colombia. [Internet]. Colombia: Centro de documentación e Información científico técnica; 2001. [revisado 2008 feb 01]. URL Disponible en: https://documentacion. ideam.gov.co/ openbiblio/Bvirtual/000001/ cap7. pdf

9. Ginther OJ. Ultrasonic imaging of equine ovarian follicles and corpora lutea. Vet Clin North Am Equine Pract 1988; 4(2):197-213.
10. Ginther OJ. Ultrasonic Imaging and Animal Reproduction: Horses. Book 2, Madison (WI): Equiservices Publishing, 1995; 73-83.

11. Ferreira-Dias G, Pinto Bravo $P$, Mateus $L$, Redmerc DA, Medeiros JA. Microvascularization and angiogenic activity of equine corpora lutea throughout the estrous cycle. Domest Anim Endocrinol 2006; 30:247-259.

12. Jablonka-Shariff A, Grazul-Bilska AT, Redmer DA, Reynolds LP. Growth and cellular proliferatin of ovine corpora lutea throughout the estrous cycle. Endocrinol 1993; 133:1871-1879.

13. Miyazaki T, Tanaka M, Miyakoshi K, Minegishi $K$, Kasai K, Yoshimura Y. Power and clour doppler ultrasonography for the evaluation of vasculature of the human corpus luteum. Reproduction 1998; 13(10):2836-2841.

14. Ginther O, Gastal E, Gastal M, Big M. Effect of prostaglandin alfa on ovarian, adrenal, and pituitary hormones and on luteal blood flow in mares. Domest Anim Endocrinol 2007; 32:315-328.

15. Townson D, Pierson R, Ginther O. Characterization of plasma progesterone concentrations for two distinct luteal morphologies in mares. Theriogenology 1989; 32(2):197-204.

16. Arruda RP, Visintin J, Fleury J, Garcia A, Madureira $E$, Celeghini E et al. Existem relações entre tamanho e morfoecogenicidade do corpo lúteo detectados pelo ultra-som e os teores de progesterona plasmática em receptoras de embriões equinos?. Braz J Vet Res Anim Sci 2001; 38:233-239.

17. Davies Morel MCG, Newcombeb JR, Swindlehurst JC. The effect of age on multiple ovulation rates, multiple pregnancy rates and embryonic vesicle diameter in the mare. Theriogenology 2005; 63:2482-2493. 